\title{
Experimental Studies of the Evaporation of Pure Liquid Droplets in a Single-Axis Non-Resonant Levitator
}

\author{
M. Radmilović-Radjenović ${ }^{1 \dagger}$, D. Radjenović ${ }^{2}$, M. Mitrić ${ }^{3}$ and B. Radjenović ${ }^{1}$ \\ ${ }^{1}$ Institute of Physics, University of Belgrade, 11080 Belgrade, Serbia \\ ${ }^{2}$ Faculty of Mathematics, University of Belgrade, 11000 Belgrade, Serbia \\ ${ }^{3}$ Vinča Institute of Nuclear Science, POB 522,1000 Belgrade, Serbia \\ †Corresponding Author Email:marija@ipb.ac.rs
}

(Received July 1, 2020; accepted September 21, 2020)

\begin{abstract}
Though a simple daily observation, evaporation of drops is still poorly understood due to the complex nature that involves hydrodynamic effects in the bulk fluids and transport phenomena at the liquid-vapor interface. This paper reports on the evaporation of single component droplets (water, ethanol, acetone, and glycerol) levitated in a single-axis non-resonant levitator. It was observed that the acetone and ethanol evaporated faster than water, although the acetone is the most volatile. The estimated lifetime of acetone is less than $5 \mathrm{~min}$, which is much shorter as compared to $56 \mathrm{~min}$ for ethanol or about $90 \mathrm{~min}$ for water droplets. On the other hand, glycerol showed no tendency to evaporate. With increasing the evaporation time, the ratio of large and small semi-axis decreases and tends to 1 corresponding to changes in drops shape from oblate ellipsoid to a sphere. Based on the classical $D^{2}$-law, the surface regression rates have been estimated.
\end{abstract}

Keywords: Evaporation; Surface regression; Pure liquid droplet; Non-resonant levitator.

\section{NOMENCLATURE}

$\mathrm{B}_{\mathrm{T}} \quad$ heat transfer number

$B_{Y} \quad$ Spalding's mass transfer number

$c_{p} \quad$ specific heat at constant pressure

$\mathrm{D}(\mathrm{t}) \quad$ instantaneous drop diameter

$\mathrm{D}_{0} \quad$ initial drop diameter

$D_{12}$ diffusion coefficient of the substance 1 in the gas 2

$\mathrm{k}$ thermal conductivity

$s_{L}(t) \quad$ length of the major semiaxes of the ellipse

$\begin{array}{ll}\mathrm{s}_{\mathrm{S}}(\mathrm{t}) & \text { length of the minor semiaxes of the ellipse } \\ \mathrm{T} & \text { temperature } \\ \mathrm{Y}_{1, \infty} & \text { mass fraction far away from the droplet } \\ \mathrm{Y}_{1, \mathrm{~s}} & \text { mass fraction on the surface } \mathrm{s} \\ \rho & \text { density of the gas mixture and the } \\ \rho_{1} & \begin{array}{l}\text { evaporated liquid } \\ \beta\end{array} \\ \tau_{\mathrm{e}} & \text { evaporation of the liquid droplet } \\ \end{array}$

ambient pressure of the vapor far away from the drop (Cachile et al. 2002; Radmilović-Radjenović et al. 2018; Brutin and Starove 2018; Sellami et al. 2019; Niimura and Hasegawa 2019; Majhy and Sen 2020). Droplet evaporation is one of the most common instances of multiphase flow with phase change, encountered in nature as well as in technical and industrial applications (Rao et al. 2010; Laux et al. 2016; Qubeissi and Sazhin 2017; Zang et al. 2019). The basic understanding of heat and mass transfer is very important for the optimization of spray drying designs. The observation of a single component
Although models and theories of droplet evaporations have been developed for over 100 years, there is still a lack of experimental data. Fundamental studies on liquid droplet evaporation started with the evaporation of water, nitrobenzene, and aniline droplets suspended in air and derivation of a well-knowThe term evaporation of droplet refers to a gradual decrease in the liquid volume. It is driven by a diffusive flux due to the difference in the vapor pressure immediately above a droplet surface and the 
droplet upon evaporation enables researchers to track all the physical and morphological changes (Zaitone 2009; Chaudhuri et al. 2020).

$\mathrm{n}$ relationship for the Sherwood number as a function of the Reynolds and Schmidt number (Ranz and Marshall 1952a). Ranz and Marshall performed experiments on the evaporation of pure component droplets with diameters ranged from 0.6 to $1.1 \mathrm{~mm}$ (Ranz and Marshall 1952a,b). Downing used the same experimental techniques as Ranz and Marshall and investigated droplet of pure liquids at temperatures from 300 to 613K (Downing 1966). Beard and Pruppacher carried out measurements of small water drops falling freely at terminal velocity in a wind tunnel (Nishimura et al. 2018). Ward and Stanga (Ward and Stanga 2001) performed experiments of water evaporation and determined the temperature profile of water and its vapor during evaporation. With the emergence of lasers, new measurement techniques evolved which allowed the determination of the droplet size with higher accuracy than by photographic imaging (Sadafi et al. 2016).

Among various diverse methods developed for the droplet evaporation, acoustic levitation is recognized as a promising tool for contactless guiding and nontoxic manipulation (Kazemi et al. 2017; Perez et al. 2019; Jacquot-Kielar et al. 2020). Levitated liquid droplets do not need to be held in a receptacle, providing benefits in accurate measurements since small objects can be suspended with the help of a standing acoustic wave. The pressure difference between two poles of the objects counteracts the gravity and acoustic streaming has a strong effect on the aspect ratio and vaporization characteristics of a suspended droplet (Tuckermann et al. 2002; Schiffter and Lee 2007; Zaitone 2018). Despite its advantages as an experimental tool, the levitator also introduces some difficulties. One of the critical issues are that the acoustic field affects the heat and mass transfer by inducing the so-called acoustic streaming, with the presence of two steady toroidal vortices close to the droplet surface. Yarin and coworkers showed that the acoustic streaming in the gas provides a convective mechanism much stronger than the ordinary one and thus dominates evaporation (Yarin et al. 1999, 2002). However, there is no theoretical model that encompasses all details included in the evaporation process.

This paper presents experimental results for the time dependence volume and the surface regression rate during evaporation of levitated single component drops composed of water, ethanol, acetone, and glycerol. Measurements were performed by using Tiny Lev acoustic levitator that allows a contactless positioning of a sample near a pressure node of a standing acoustic wave emitted by 72 simple ultrasonic emitters. (Marzo et al. 2017). It was demonstrated that liquid evaporation during acoustic levitation can be studied by using a relatively simple experimental set-up than can be built by using lowcost components. Based on the fit of the experimental results, the speed of evaporation and the surface regression rate has been estimated. It was shown that the acetone evaporates the fastest with the shortest lifetime, while the glycerol does not evaporate at all. For all droplets, as time increased, aspect ratios of droplets of water, acetone, and ethanol approach to 1 .

\section{THEORETICAL BACKGROUND}

The evaporation of pure liquid droplets are usually described by simple evaporation models based on the film theory and illustrated in Fig. 1 (detailed description is given in Zaitone 2009). According to film theory, heat is transported solely by diffusion towards the droplet in exchange for mass diffusion from the evaporating droplet in the opposite direction. The film is surrounded by two boundaries: the inner boundary indefinitely close to the droplet surface is denoted by the subscript $s$, while the subscript $\infty$ corresponds to the outer boundary i.e. conditions far away from the droplet. The dominant parameters for heat and mass transfer are the temperature $T$ and the mass fraction of the fuel $Y$. Between the droplet surface and the surface $s$, the phase change from liquid-phase to gas-phase quantities takes place. From the droplet surface to the surface $s$, the temperature remains constant, but the mass concentration changes abruptly. The mass transfer rate is defined as the mass flow rate per unit area $A$ at the droplet surface (Zaitone 2009):

$$
\dot{\mathrm{m}}^{\prime \prime}=\frac{\dot{\mathrm{m}}}{\mathrm{A}}=\frac{2 \rho \mathrm{D}_{12}}{\mathrm{D}} \ln \left(1+\mathrm{B}_{\mathrm{Y}}\right),
$$

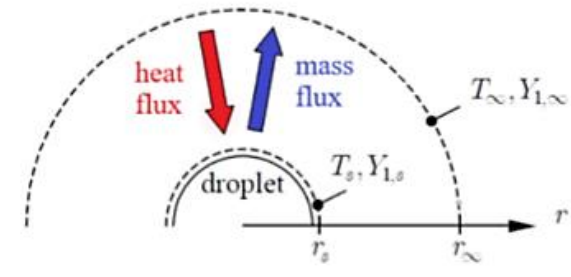

1. Schematic view of the film model for the droplet evaporation (more details can be found in Ref. Zaitone 2009).

where is the droplet diameter, $\rho$ is the density of the mixture of the gas and the evaporated liquid, $D_{12}$ is the diffusion coefficient of the evaporated liquid as substance 1 in the gas with index 2, while Spalding's mass transfer number $B_{Y}$ is expressed as (Zaitone 2009):

$$
\mathrm{B}_{\mathrm{Y}}=\frac{\mathrm{Y}_{1, \infty}-\mathrm{Y}_{1, \mathrm{~s}}}{\mathrm{Y}_{1, \mathrm{~s}}-1} \text {. }
$$

Combining Eqs. (1) and (2) for the mass transfer rate we obtain (Zaitone 2009):

$$
\dot{\mathrm{m}}^{\prime \prime}=\frac{2 \rho \mathrm{D}_{12}}{\mathrm{D}} \ln \left(\frac{\mathrm{Y}_{1, \infty}-1}{\mathrm{Y}_{1, \mathrm{~s}}-1}\right),
$$

where $Y_{1, s}$ and $Y_{1, \infty}$ denote the mass fraction on the surface $s$ and far away from the droplet, respectively. Equation (3) can be expressed via heat transfer number $B_{T}$ (Zaitone 2009): 
$\dot{\mathrm{m}}^{\prime \prime}=\frac{2 \mathrm{k}}{\mathrm{c}_{\mathrm{p} 1} \mathrm{D}} \ln \left(1+\mathrm{B}_{\mathrm{T}}\right)$

with thermal conductivity $k$ and the specific heat at constant pressure $c_{p}$. If the expression for the Spalding's number (Zaitone 2009):

$\mathrm{B}_{\mathrm{T}}=\frac{\mathrm{c}_{\mathrm{p} 1}\left(\mathrm{~T}_{\infty}-\mathrm{T}_{\mathrm{s}}\right)}{\Delta \mathrm{h}_{\mathrm{v}}}$,

is substituted in Eq. (4) the mass transfer rate can be expressed in terms of the unknown temperature $T_{s}$ (Zaitone 2009):

$\dot{\mathrm{m}}^{\prime \prime}=\frac{2 \mathrm{k}}{\mathrm{c}_{\mathrm{p} 1} \mathrm{D}} \ln \left(1+\frac{\mathrm{c}_{\mathrm{p} 1}\left(\mathrm{~T}_{\infty}-\mathrm{T}_{\mathrm{s}}\right)}{\Delta \mathrm{h}_{\mathrm{v}}}\right)$.

Since droplet size is changed due to the mass transfer rate the equation of mass conservation is applied to the droplet surface (Zaitone 2009):

$\dot{\mathrm{m}}^{\prime \prime}=-\frac{\dot{\mathrm{m}}}{\mathrm{A}_{\mathrm{s}}}=-\frac{1}{\pi \mathrm{D}^{2}} \frac{\mathrm{d}}{\mathrm{dt}}\left(\rho_{1} \frac{\pi}{6} \mathrm{D}^{3}\right)$,

where $\rho_{l}$ is the density of the liquid droplet. "Equation (7)," together with "Eq. (1)," leads to:

$\frac{\mathrm{dD}^{2}}{\mathrm{dt}}=-\frac{8 \rho \mathrm{D}_{12}}{\rho_{\mathrm{l}}} \ln \left(1+\mathrm{B}_{\mathrm{Y}}\right)$

with a constant right-hand side. The evaporation rate $\beta$ is expressed as (Zaitone 2009):

$\beta=-\frac{\mathrm{dD}^{2}}{\mathrm{dt}}=\frac{8 \rho \mathrm{D}_{12}}{\rho_{\mathrm{l}}} \ln \left(1+\mathrm{B}_{\mathrm{Y}}\right)$.

The evolution of the droplet diameter can be obtained by integrating equation Eq. (8) with the boundary condition $D(t=0)=D_{0}($ Quino et al.2015):

$D^{2}(t)=D_{0}^{2}-\beta t$, or

$\frac{\mathrm{D}^{2}(\mathrm{t})}{\mathrm{D}_{0}^{2}}=1-\beta \frac{\mathrm{t}}{\mathrm{D}_{0}^{2}}$, or

well known as $D^{2}$-law, where $D_{0}$ and $D(t)$ are the initial and instantaneous drop diameters in $\mathrm{mm}$, respectively, $t$ is time in seconds and $\beta$ is the instantaneous surface regression rate. According to $D^{2}$-law square of the droplet diameter decreases linearly with time during droplet evaporation. The prediction of droplet diameter evolution by $D^{2}$-law represents a classic way to model droplet evaporation not taking into account the evaporation of droplet by forced convection, natural convection or even under the influence of the acoustic filed (Zaitone 2009). Since the mass transfer rate can be expressed either in terms of quantities from mass diffusion or heat diffusion, the evaporation rate coefficient $\beta$ can be also written using heat transfer quantities (Zaitone 2009):

$\beta=\frac{8 \mathrm{k}}{\rho_{1} \mathrm{c}_{\mathrm{p} 1}} \ln \left(1+\mathrm{B}_{\mathrm{Y}}\right)$.

The validity of expression (12) can be checked by plotting the evaporation data $\left(D / D_{0}\right)^{2}$ versus $t / D_{0}^{2}$ and confirming that the resulting evaporation curve is linear from whose slope $\beta$ can be determined. Deviations from the $D^{2}$-law can be seen as deviations from a straight line. The lifetime $\tau_{e}$ of the droplet is another important parameter in droplet evaporation determined from eq. (10) for $D\left(t=\tau_{e}\right)=0$ (Zaitone 2009):

$\tau_{\mathrm{e}}=\mathrm{D}_{0}^{2} / \beta$.

\section{EXPERIMENTAL TECHNIQUE}

A schematic view of the experimental setup used in this study is shown in Fig. 2. Droplets were levitated by a single-axis non-resonant levitator made with off-the-shelf low-cost components. The main components of the levitator are 72 transducers that transform the electrical input signal generated by an Arduino Nano into acoustic wavers. In TinyLev, Ningbo or Manorshi 10mm transducers are used, and they reduce only $3 \%$ in trapping force due to their phase deviation. Since acoustic waves at the operation frequency of transducers of $40 \mathrm{kHz}$ have a wavelength of $8.65 \mathrm{~mm}$, TinyLev levitator allows the levitation of samples of up to $\approx 4 \mathrm{~mm}$ (halfwavelength). Detailed description on Tiny Lev acoustic levitator is provided in reference (Marzo et al. 2017).

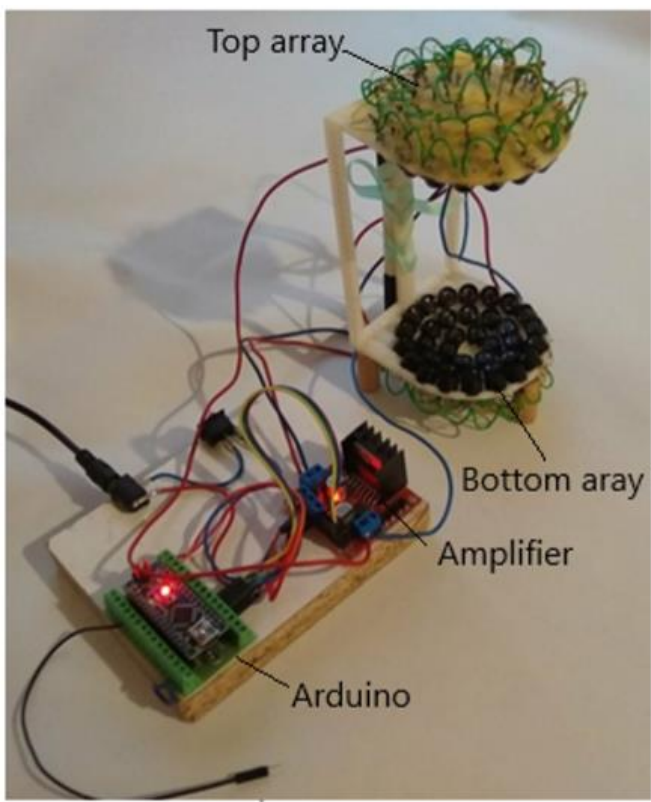

Fig. 2. Experimental set-up composed of the driver board and the single axis levitator with 72 transducer operating ate $40 \mathrm{kHz}$ (more details in Marzo et al. 2017.)

Measurements were performed for pure liquid droplets: water, ethanol, acetone, and glycerol. Experimental conditions include ambient pressure and the ambient temperature in the range from $20^{\circ} \mathrm{C}$ to $23^{\circ} \mathrm{C}$. For the formation of evaporating drops, liquids were delivered manually through a capillary into one of the node points of the acoustic levitator 
as depicted in Figure 3a that shows two droplets are levitated at the same time at two different acoustic nodes. The upper droplet denoted as a control droplet is polystyrene ball with known dimensions. The lower droplet is the pure liquid droplet under observation. During measurements, the bottom part of the levitator is covered by gauze in order to protect it from wetting as shown in Fig. 3b. For each drop 5 sets of measurements were recorded using the smartphone camera with the resolution of 16megapixels. Then, for a particular time interval, frames are extracted by programm Moviemaker (Birney et al. 2000) and then the dimension of the droplet is determined by comparison the dimension of the control droplet. The meridian contour of the droplet is approximated by an ellipse, which has proved to be always a correct approximation. As can be seen from Fig. 4, if the ellipse is rotated about a minor axis, an equator is formed at the endpoints of the major axis and an oblate is obtained (Zaitone 2018). Based on the oblate shape of the droplet, its volume is calculated assuming axis symmetry. The droplet diameter, vertical position and the aspect ratio are monitored as a function of time during the droplet evaporation from extracted frames opened in Paint. The accuracy of the approximation of the drop contour by the ellipse is better than $2 \%$ in the representation of the major and minor semiaxes. The matching volume equivalent diameter is calculated as (Hilbert and Cohn-Vossen 1999):

$$
D(t)=2 \sqrt[3]{s_{L}(t)^{2} \cdot s_{S}(t)}
$$

where $s_{L}(t)$ and $s_{S}(t)$ are the lengths of the major and minor semiaxes of the ellipse. a)

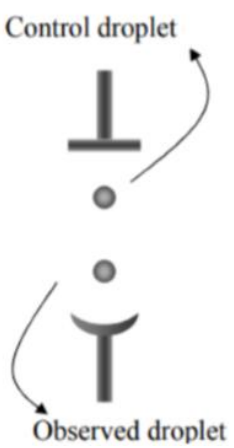

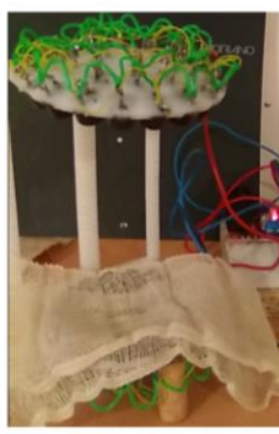

Fig. 3. a) Sketch and b) snapshot of two droplet levitated in the acoustic field.
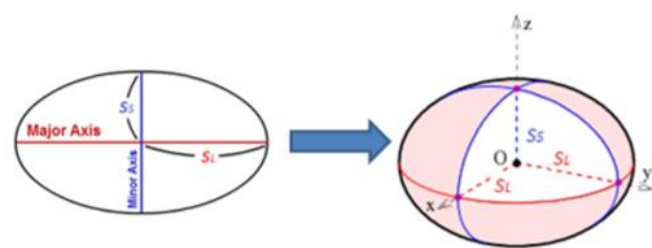

Fig. 4. Oblate ellipsoid formed by revolving ellipse about a minor axis when an equator is

formed at the endpoints of the major axis and an oblate is obtained (Hilbert and Cohn-Vossen 1999).

\section{RESUlTS}

Results of test measurements performed for $1.5 \mu 1$ water droplets are shown in Fig. 5. The droplet volume decreases with time, as expected. Our results (red symbols) agree well with the data taken from the literature (Knutsson 2006) (light blue symbols). Both results demonstrate that the evaporation process is not a completely linear function. Blue lines correspond to the regression lines showing the linearity of the evaporation at different stages of the evaporation process. In the beginning, the evaporation curve is steeper which means a higher evaporation rate than the rest of the curve. Since evaporation takes place at the surface of the droplet, the process is dependent on the surface area of the droplet and thereby on the volume. The larger the surface area leads to the faster evaporation of the droplet. For volume changes from $1.5 \mu 1$ down to $1 \mu 1$ evaporation speed is $2.5 \cdot 10^{-3} \mu \mathrm{l} / \mathrm{s}$. Overall evaporation speed is $10^{-3} \mu 1 / \mathrm{s}$.

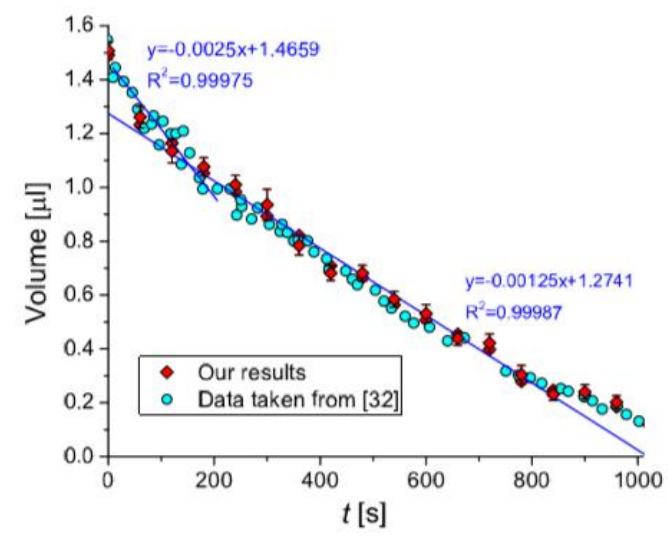

Fig. 5. The volume as a function of time for $1.5 \mu$ water droplet. Our results are shown by red symbols, while light blue symbols are data from the literature (Knutsson 2006).

Figure 6 contains results for decreasing the droplet volume with time during the evaporation. As can be observed, the acetone evaporates very fast and there is no more droplet after no more than $300 \mathrm{~s}$. The evaporation of ethanol is lower than that for acetone but faster than that for the water. On the other hand, glycerol droplets show no tendency to evaporate (Knutsson 2006). To obtain the speed of evaporation the evaporation graphs shown in Fig. 6 are fitted by the regression lines and the obtained values are listed in Table 1.

Table 1 Data for the pure liquids samples in the evaporation experiments shown in Fig. 6 and Fig. 7

\begin{tabular}{|c|c|c|c|}
\hline & Water & Ethanol & Acetone \\
\hline $\begin{array}{c}\text { Speed of evaporation } \\
{[\mu \mathrm{l} / \mathrm{s}] \times 10^{-4}}\end{array}$ & $9.0 \pm 0.4$ & $12 \pm 1$ & $83 \pm 3$ \\
\hline $\begin{array}{c}\text { Evaporation } \\
\text { rate }\left[\mathrm{mm}^{2} / \mathrm{s}\right] \times 10^{-4}\end{array}$ & $6.8 \pm 0.2$ & $11 \pm 1$ & $97 \pm 8$ \\
\hline
\end{tabular}




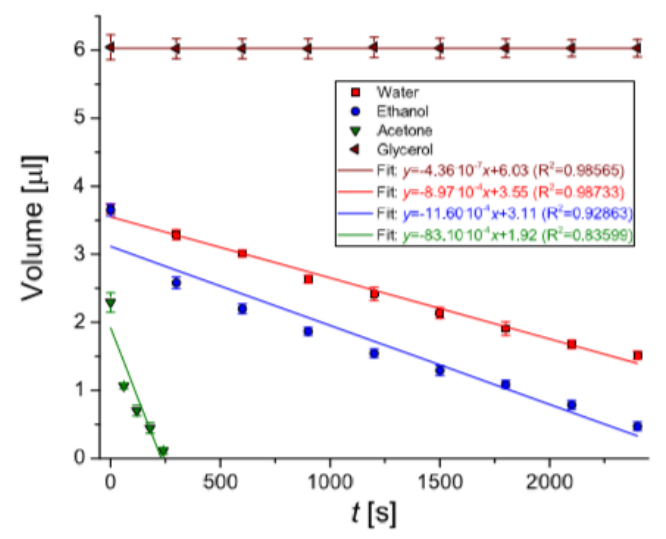

Fig. 6. The time dependence of the volume of pure liquids droplets. Results of measurements are presented by symbols, while lines correspond to the linear regression fits.

Figure 7 shows $D^{2}(t) / D_{0}^{2}$ versus $t / D_{0}^{2}$ for the water, acetone, ethanol and glycerol droplets. Results of measurements (solid symbols) have the similar trends as theoretical predictions based on Eq. (11) (open symbols), albeit systematically higher. The lines result from fits of Eq. (11) to the data points. The obtained values for the surface regression rate are also listed in Table 1. As expected, for a single component drops the surface regression rate is a constant (Quino et al. 2015). It would be more illustrative by estimating the droplet lifetime by Eq. (13). From our measurements, we obtain values of around: $90 \mathrm{~min}$, 56min and $3 \mathrm{~min}$ as the lifetime of water, ethanol and acetone droplet, respectively.

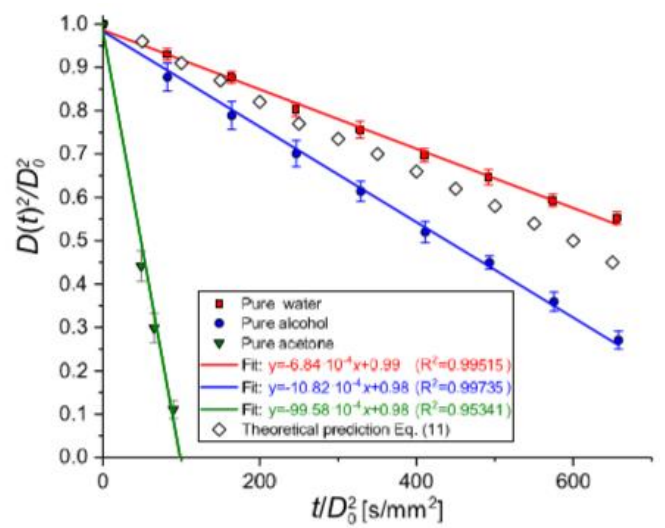

Fig. 7. $D^{2}(t) / D_{0}^{2}$ as a function of $t / D_{0}^{2}$ for pure liquids droplets. Symbols and lines correspond to the experimental data and the linear regression fits, respectively.

There is an important concept of aspect ratio defined as the ratio of the lengths of the major and minor semiaxes of the fitted oblate ellipse which is associated with geometric shapes. The aspect ratio as a function of time is depicted in Fig. 8. With increasing time, the aspect ratio of droplets of all considered pure liquids, excepted glycerol, decreases and approaches 1 corresponding to the changes in the shape of the drops from oblate ellipsoid to a sphere as illustrated in Fig. 9. It could be attributed to the fact that the levitation itself causes non-negligible forces on the drops surface which, according to (Brenn et al. 2007), can induce convection inside the drop. If the droplet becomes smaller, the surface tension force becomes more dominant than the acoustic radiation stress and the droplet gets more spherical. Since glycerol droplet does not evaporate, its aspect ratio does not change during the time.

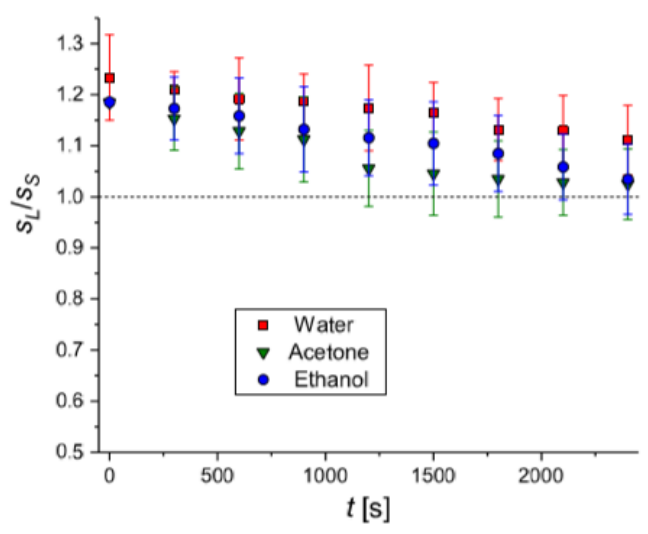

Fig. 8. The time dependence of the aspect ratio (the ratio of the lengths of the major and minor semiaxes of the fitted oblate ellipse) of pure liquids droplets.

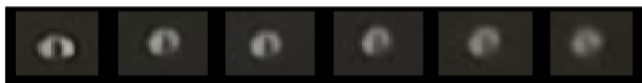

Fig. 9. The time evolution of the water droplet from the oblate ellipsoid to a sphere.

Finally, if we apply Eq. (12) to the measured data, we may estimate the lifetime $\tau_{e}$ of a droplet as listed in Table 2. As can be expected, the lifetime of the acetone droplet is less than $5 \mathrm{~min}$, while the droplet made of water and ethanol may levitate around $90 \mathrm{~min}$ and $55 \mathrm{~min}$, respectively.

Table 2. Lifetimes of a droplets composed of water, ethanol, and acetone determined according to Eq. (12) and the data shown in Fig.

\begin{tabular}{|c|c|c|c|}
\hline \multicolumn{4}{|c}{8 and Table 1. } \\
\hline & Water & Ethanol & Acetone \\
\hline$\tau_{\mathrm{e}}[\mathrm{s}]$ & $5260 \pm 70$ & $3310 \pm 50$ & $276 \pm 8$ \\
\hline
\end{tabular}

\section{CONCLUSIONS}

This paper contains results which clearly demonstrate how relatively simple experimental setup can be used for measurements of the evaporation and the surface regression rates of pure liquid droplets in an acoustic levitation device. A single-axis nonresonant levitator is built by using low-cost components following the description in Ref. Marzo et al. 2017. A good agreement between test measurements for $1.5 \mu \mathrm{m}$ water droplets and the 
experimental data taken from the literature (Knutsson 2006) confirms the accuracy of the method. Measurements were also performed for water, ethanol, acetone, and glycerol under the ambient temperature and pressure. The timedependent volume of the droplet and $D^{2}(t) / D_{0}^{2}$ as a function of $t / D_{0}^{2}$ are plotted. It was observed that the evaporation process is not a completely linear function. Actually, evaporation depends on the volume and therefore on the surface, so the larger surface area leads to the faster evaporation of the droplet. The shrinkage of drops can be described by the $D^{2}$-law. Based on the fit of the experimental data, the speed of evaporation and the surface regression rate is estimated. In line with the expectations, ethanol and acetone evaporate much faster than water and glycerol, although the acetone is more volatile. The lifetime of acetone droplet is around $5 \mathrm{~min}$ and is much shorter than $56 \mathrm{~min}$ and $90 \mathrm{~min}$ as the lifetime of ethanol and water, respectively. On the other hand, it was observed that glycerol drop is not subject to the evaporation. During evaporation, the aspect ratio of droplets tends to 1 which is followed by the transformation of the shape of the drops from oblate ellipsoid to a sphere. Based on the presented results and their good agreement with the data from the literature one may conclude that acoustic levitation is a very powerful tool that can be used not only in research but also in school laboratories to process liquid materials without the presence of surfaces.

\section{ACKNOWLEDGMENTS}

The authors acknowledge funding provided by the Institute of Physics Belgrade, through the grant by the Ministry of Education, Science and Technological Development of the Republic of Serbia. The author DR also acknowledges support by Faculty of Mathematics, University of Belgrade.

\section{REFERENCES}

Birney, B., M. Lichtenberg and S. McEvoy, S. (2000). Microsoft Windows Movie Maker handbook. Microsoft Press, Chicago, USA.

Brenn, G., L. J. Deviprasath, F. Durst and C. Fink (2007). Evaporation of acoustically levitated multi-component liquid droplets. Int. J. Heat Mass Transfer 50(2526), 5073-5086.

Brutin, D. and V. Starovc (2018). Recent advances in droplet wetting and evaporation. Chemical Society Reviews 47(2), 558-585.

Cachile, M., O. Benichou, C. Poulard and A. M. Cazabat (2002). Evaporating Droplets. Langmuir 18(21), 8070-8078.

Chaudhuri, S., S. Basu, P. Kabi, V. R. Unni and A. Saha (2020). Modeling the role of respiratory droplets in Covid-19 type pandemics. Physics of Fluids 32(6), 063309.

Downing, C. G. (1966). The evaporation of drops of pure liquids at elevated temperatures: rates of evaporation and wet-bulb temperatures. AIChE Journal 12(4), 760-766.

Hilbert, D. and S. Cohn-Vossen (1999). Geometry and the Imagination. Chelsea, New York.

Jacquot-Kielar, J., M. Talbi and M. Brunel (2020). Third glare point effect in 90 Interferometric Laser Imaging for Droplet Sizing. Optics Communications 462(1), 125349.

Kazemi, M. A., D. S. Nobes and J. A. W. Elliott (2017). Experimental and Numerical Study of the Evaporation of Water at Low Pressures. Langmuir 33(18), 45784591.

Knutsson, M. (2006) Acoustic Levitation Optimization of instrumental parameters of the LevMac instrument for protein crystallization applications. Bc Thesis, Lunds University, Linds, Sweden.

Laux, D., J. Y. Ferrandis and D. Brutin (2016) Ultrasonic monitoring of droplets evaporation: Application to human whole blood. Ultrasonics Sonochemistry 32(9), 132-136.

Majhy, B. and A. K. Sen (2020).Evaporationinduced transport of a pure aqueous droplet by an aqueous mixture droplet. Physics of Fluids 32(3), 032003

Marzo, A., A. Barnes and B. W. Drinkwater (2017). TinyLev: A multi-emitter singleaxis acoustic levitator. Review of Scientific Instruments 88(10), 085105(1-6).

Niimura, Y. and K. Hasegawa (2019). Evaporation of droplet in mid-air: Pure and binary droplets in single-axis acoustic levitator. PLoS ONE 14(2), e0212074.

Nishimura, A., H. Weller, H. Maruoka, M. Takayanagi and H. Ushiki (2018). Dynamics of a dry-rebounding drop: observations, simulations, and modeling. Open Physics 16(1), 271-284

Perez, A. T., P. Garca-Snchez, M. A. S. Quintanilla, and A. Fernndez-Prieto (2019). Levitation? Yes, it is possible!. American Journal of Physic 87(4), 270-274.

Qubeissi, M. A. and S. S. Sazhin (2017). Models for droplet heating and evaporation: an application to biodiesel, diesel and gasoline fuel. International Journal of Engineering Systems Modelling and Simulation 9(1), 32-40.

Quino, J., T. Hellwig, M. Griesing, W. Pauer, H. U. Moritz, S. Will and A. Braeuer (2015). Onedimensional Raman spectroscopy and shadowgraphy for the analysis of the evaporation behavior of acetone/water drops. International Journal of Heat and Mass Transfer 89(10), 406-413.

Radmilović Radjenović M., D. Radjenović, B. Stojičić and B. Radjenović (2018). Experimental studies of the evaporation of the spheroidal oblate water droplets in a single-axis non-resonant levitator. International Journal 
M. Radmilovic-Radjenovic et al. / JAFM, Vol. 14, No. 2, pp. 581-587, 2021.

for Innovative Research in Multydisciplinary Field 4(9), 55-59.

Ranz, W. E. and W. R. Marshall Jr (1952a). Evaporation from drops: Part I. Chemical Engineering Progress 48(3), 141-146.

Ranz, W. E. and W. R. Marshall Jr (1952b). Evaporation from drops: Part II. Chemical Engineering Progress 48(3), 173-180.

Rao, N., N. Kadrichu and B. Ament (2010). Application of a droplet evaporation model to aerodynamic size measurement of drug aerosols generated by a vibrating mesh nebulizer. Journal of Aerosol Medicine and Pulmonary Drug Delivery 23(5), 295-302.

Sadafi, M. H., S. Gonzlez Ruiz, M. R. Vetrano, van Beeck, I. Jahn, J. M. Buchlin and J. Hooman (2016). On the Influence of Low-power Laser Source on the Evaporation of Single Droplets: Experimental and Numerical Approaches. Journal of Applied Fluid Mechanics 9(0), 8187.

Schiffter, H. and G. Lee (2007). SingleDroplet Evaporation Kinetics and Particle Formation in an Acoustic Levitator. Part 1: Evaporation of Water Microdroplets Assessed Using Boundary-Layer and Acoustic Levitation Theories. Journal of Pharmaceutical Sciences 96(9), 22742283

Sellami, K., N. Labsi, M. Feddaoui, M. Oubella, Y. K. Benkahla, M. Najim (2019). Comparative Study of Reversal Flow during the Evaporation or Condensation of Water and Ethanol Film in a
Vertical Channel. Journal of Applied Fluid Mechanics 11(5), 1421-1426.

Tuckermann, R., S. Bauerecker and B. Neidhart (2002). Evaporation rates of alkanes and alkanols from acoustically levitated drops. Analytical and Bioanalytical Chemistry 372(12), 122-127.

Ward, C. A. and D. Stanga (2001). Interfacial conditions during evaporation or condensation of water. Physical Review E 64(10), 051509 (19).

Yarin, A. L., G. Brenn, O. Kastner, D. Rensink and C. Tropea (1999). Evaporation of acoustically levitated droplets. Journal of Fluid Mechanics 399(11), 151-204.

Yarin, A. L., G. Brenn and D. Rensink (2002). Evaporation of acoustically levitated droplets of binary liquid mixtures. International Journal of Heat and Fluid Flow 23(4), 471-486.

Zaitone, B. A. (2009). Drying of Multiphase Single Droplets in Ultrasonic Levitator. $\mathrm{PhD}$ Thesis, Technische Universitt Darmstadt, DE.

Zaitone, B. A. (2018). Oblate spheroidal droplet evaporation in an acoustic levitator. International Journal of Heat and Mass Transfer 126(Part B), 164-172.

Zang, D., S. Tarafdar, Y. Yu. Tarasevich, M. D. Choudhury and T. Dutta (2019). Evaporation of a Droplet: From physics to applications. Physics Reports 804(4), 1-56. 